\title{
Egalitarianism: Psychological and socio-ecological foundations
}

\author{
${ }^{*}$ Jennifer Sheehy-Skeffington ${ }^{1}$ \& Lotte Thomsen ${ }^{2,3}$
}

${ }^{1}$ London School of Economics and Political Science, Houghton Street, London WC2A 2AE, London U.K.. Contact: j.a.sheehy-skeffington@lse.ac.uk (corresponding author)

2 University of Oslo, Problemveien 7, 0315 Oslo, Norway. Contact: lotte.thomsen@psykologi.uio.no

${ }^{3}$ Aarhus University, Bartholins Allé 7, DK-8000 Aarhus C, Denmark.

Individual differences in social and political attitudes have their roots in evolved motives for basic kinds of social relationships. Egalitarianism is the preference for the application of the one of these relational modelsequality-over that of another-dominance-to the context of societal intergroup relations. We present recent research on the origins of egalitarianism in terms of universal social cognitive mechanisms (activated as early as infancy), systematic (partly heritable) individual differences, and the affordances and constraints of one's immediate and macro-structural context. Just as the psychological impact of socioeconomic conditions depends on the mind being equipped to perceive and navigate them, so the expression of the evolved underpinnings of inequality concerns depends critically on social and societal experiences.

Highlights:

- Societal politics and ideology are undergirded by basic relational strategies rooted in dilemmas of resource distribution.

- Core representations and motives for equality and hierarchy emerge in infancy.

- Individual differences in egalitarian strategies manifest in infancy, stabilize in adolescence, and are partly heritable.

- The extent, consensuality, and predictive power of egalitarianism depend on societal context.

- A multi-level approach is needed to account for both the biological and socio-ecological foundations of egalitarianism.

Key words: egalitarianism; social dominance orientation; relational models theory; ideology; equality; hierarchy

\section{Introduction}

A central dilemma at the interface of the individual and the socio-ecological context concerns the distribution of resources, whether assets, territory and living conditions, or rights such as priority, preferential care, and decision-making. Such material and political arrangements and the relationships they engender are so fundamental to the challenge of human sociality that the psychology for navigating them has deep evolutionary, developmental, and cross-cultural roots [1,2]. We argue that attitudes toward the (un)equal distribution of resources in society are grounded in universal representations and motives concerning basic social relationships, whose manifestation is critically dependent on socio-ecological context. Individual levels of egalitarianism at any one point in time reflect ongoing interactions between evolved psychological architecture, systematic individual differences, and the affordances and constraints of one's immediate and macro-structural environment.

\section{Navigating inequality is core to social cognition}

In order for socio-ecological conditions to have a formative influence on human psychology, the human mind must arrive in the world with the ability to understand and respond to social relationships that have a political and material dimension [3,4]. Indeed, evidence from multiple sources indicates that representations and preferences concerning unequal social relations constitute the building blocks of social cognition, with downstream consequences for perceptions, attitudes, and behavior.

\footnotetext{
${ }^{*}$ Both authors contributed equally to this work.
} 
Inequality concerns are at the core of two of the four basic relational forms which have been found to govern resource distribution across cultures. A relationship based on equality determines that resources be balanced and distributed to everyone to the same degree and/or in turns, whereas one based on hierarchy determines that they be allocated preferentially to those at the top in terms of dominance, authority, or prestige [1]. Distributive fairness, on this account, does not equate to equality, but is instead the satisfactory enactment of the rules of a particular relational model in its appropriate social context.

Representations and even motives concerning the principles of equality and hierarchy emerge early in infancy, suggesting they form part of the set of innate social-cognitive mechanisms with which all humans are endowed. Preverbal infants appear egalitarian, predicting and preferring that outcomes be distributed equally in the absence of other information [5-9], and approaching equal over unequal distributors [7,8]. If one agent has previously dominated another, however, they expect third parties will distribute most resources to the more dominant agent [10] (see also [11]). Infants also use the formidability cues of body, coalition size, and previous win-lose history to predict who will dominate whom in zero-sum conflict [12-14], and those who prevail because others defer to them motivate selective affiliation behavior [15]. Although in principle egalitarian expectations need not imply altruistic sharing of personal, valuable resources (cf. [1]), systematic individual differences that functionally link the two seem to emerge in infancy: A significant majority of the infants who expect equal resource distributions also share their preferred toy with an unfamiliar experimenter, whereas most infants who expect unequal distributions keep their preferred toy to themselves $[9,16,17]$. Importantly, this link between egalitarianism and altruism is already parochial in nature, with children's desire to ensure equal outcomes being limited to ingroup members, once group identity is made salient [18] (see also [19]).

As people move from childhood to adulthood, they face the critical question of how resources should be distributed in a wider collective: Is it fair or unfair that some groups of people have more resources than others, and if so, when and through what principles? We argue that egalitarianism may be scaled from the interpersonal to the intergroup level, as a preference that the basic relational form of equality, as opposed to that of hierarchy, govern the distribution of resources in a society. Indeed, the most widely used measure of anti-egalitarianism, social dominance orientation (SDO) [20], consists of two sub-dimensions corresponding to both equality and hierarchy: SDO-E measures preferences regarding equality between social groups, while SDO-D indexes an orientation in favor of groups being arrayed in a dominance hierarchy [21,22].

Decades of research conducted in multiple countries and cultures demonstrates that anti-egalitarianism measured in this way predicts support for ideologies, policies and behaviours that enhance versus attenuate disparities between social groups, including racism, sexism, fiscal conservatism, and harsh treatment of the poor, criminals, and immigrants $[2,23,24]$. SDO has emerged as a key explanatory variable in understanding even the most recent efforts to uphold intergroup hierarchy, such as through far-right nationalism and persecution of Muslims [25-29] (see also [30]), suggesting that such efforts are fueled by equality concerns that are ultimately grounded in dilemmas of resource distribution. This orientation is not only general, but enduring, and influential: new work using advanced longitudinal methods has demonstrated the stability of both sub-dimensions of SDO, as well as its ability to predict other social attitudes over time, already in adolescence [31] (see also [32,33]).

Importantly, key findings among adults at the intergroup level mirror those among infants and young children at the interpersonal level: general expectations that resources should be distributed equally correlate with being willing to help and share resources with others, specifically, low power societal groups [24]. For example, egalitarianism predicts willingness to pay taxes to fund government-provided universal healthcare across 29 nations [34]. Echoing default infant expectations and preferences for equality, average levels of SDO in representative population samples and meta-analytic estimates of the average SDO level across many nations indicate that the majority of adults prefer between-group equality over hierarchy (see [35-37]). And to the degree that adults prefer between-group hierarchy, they endorse legitimizing myths that justify such unequal resource distributions as the product of right and fair commonly-recognized principles (e.g., conservatism, the Protestant Work Ethic, or meritocracy [2]), not by appeal to crude differences of power [2], echoing the way toddlers approach those whose precedence is recognized by others, but not those who retort to brute coercion [15]. 


\section{Individual differences in egalitarianism reflect flexible biological strategies that respond to social ecology}

We have seen one way in which socio-ecological concerns are inscribed in human nature, through universal, early emerging representations and motives concerning equality and hierarchy, which arguably underpin egalitarianism at the intergroup level. Might it also be possible that individual differences in the application of equality versus hierarchy to societal resource distribution, too, are reflected in such evolutionary logic? The potentially evolved nature of the social cognitive processes underlying egalitarianism might lead one to expect little of the individual variation in their workings to have biological roots [38]. Yet, precisely because of the changing nature of socio-ecological demands and affordances, there are also reasons why evolution might sustain heritable variation in dispositions toward egalitarian versus hierarchical strategies.

For example, the evolutionary logic of social bargaining leads to the prediction that those with greater ability to win fights over resources, insofar as they will be more likely to end up near the top of social hierarchies, will give greater support to unequal resource distributions. In support of this claim, multiple studies from international samples report a positive correlation between SDO (and related forms of anti-egalitarianism) and physical formidability, in particular among men, for whom resources have greatest adaptive value [39-42]. This association holds even when controlling for time spent on activities designed to increase formidability (i.e. lifting weights), suggesting that physical characteristics drive SDO levels, rather than the reverse [42]. However, the relationship is also shaped by socio-ecological cues, as it comes out most strongly among those men who have achieved a dominant hierarchy position in terms of socioeconomic standing $[40,42]$.

Thus, to the extent that egalitarianism is partly heritable, its transmission is likely governed by principles of balancing or fluctuating selection, in which the adaptive value of a trait depends on the dynamics of social ecology (including the existing distribution of related traits in a population, see $[43,44]$ ). Evolutionary game theoretical modelling based on these principles leads to the expectation that any population will possess a variety of heritable adaptive strategies for navigating equal and hierarchical social relations, analogous to the stabilization of hawk/dove dynamics (see [45]). Consistent with this, the largest twin study conducted to date found that both the equality and the hierarchy sub-dimensions of SDO have substantial heritability ( $24 \%$ and $37 \%$, respectively). Importantly, the shared variance between SDO and political attitudes predominantly reflected the same genetic, rather than environmental, underpinnings (mean genetic correlation 0.51) [46]. This implies that the adaptive strategies being passed on from one generation to the next involve differing preferences for equality versus hierarchy to apply to societal intergroup relations in addition to the proclivity to implement these relational preferences through whatever hierarchy-attenuating versus hierarchy-enhancing means are afforded by the concurrent political, cultural, social, and material context-a 'behavioural syndrome' for resource distribution and territoriality, to use the cross-species language of behavioural ecology [47]. Convergent with this, adolescents resemble their parents in general anti-egalitarianism, as captured by SDO, which in turn mediates parent-child similarity in general prejudice [48].

\section{Egalitarianism responds to societal conditions}

A key insight of the socio-ecological approach is that any evolved psychological proclivities and their genetic variation must be realized in the context of relationships and environments strewn with objective power and resource differentials. Indeed, although we recently found SDO to be heritable, the strongest setting of SDO levels in fact occurred through the unique environmental experiences of each individual twin [46], highlighting the centrality of socio-ecological conditions in determining one's level of egalitarianism. At the interpersonal level, which relational form will stabilize to govern the distribution of resources depends on the possibility, costs and benefits of acquiring, monopolizing and consuming resources for a particular individual in a particular context (cf. [49]). At the intergroup level, whether it is adaptive to desire the application of equality versus hierarchy to societal relations should thus depend not only one one's individual characteristics and social experiences, but also the nature of the broader social-structural context and one's position within it (see also [50]).

Indeed, in line with social dominance theory, levels of SDO are affected by the experience of being socialized or temporarily placed into a high status group (e.g., [51,52]; see also [11]), while experimental work has observed SDO to increase with situational threats to status or identity (e.g., most recently, [53]). 
Research is currently turning toward influences on processes of egalitarianism at the societal level, the most relevant socio-ecological context for adjudicating intergroup resource distribution. Cross-national differences in average levels of egalitarianism are observed as early as childhood. Recent multi-nation studies using economic games have found that that children's tendency to object to inequalities between third parties, or unequal situations that benefit them, emerges earlier and more reliably in Western, individualistic cultures [54-56]. Aversion to inequality in such abstract scenarios is also observed to be higher among adults in societies with greater market integration, an index of economic development [57]. This pattern in economic game behavior is similar to the cross-societal pattern in expressed preferences for equality, which are more prevalent in states with higher gross national income [35]. On the other hand, macro-structural inequality (as measured by the GINI coefficient and indices of gender and happiness inequality) increases psychological preferences for intergroup inequality among high status groups across nations and American states, and these anti-egalitarian preferences are also associated with poorer levels of democratic governance and press freedom, greater risk of violent conflict, and greater intergroup discrimination $[35,58]$. Thus, it seems that it pays off, in general, for people to endorse inequality at the interpersonal and intergroup level if they occupy high power positions in already unequal contexts in which resources are scarce, identity is a source of conflict, and intergroup repression is the norm. Indicating that social-ecological dynamics in part play out through evolved behavioral syndromes that manifest at the individual level (and that any such psychological behavioral syndromes are critically affected by socio-ecological dynamics), the effect of macro-structural economic inequality on support for racism, sexism, persecution of immigrants, and opposition to social welfare among White Americans surveyed across American states was mediated by individual support for anti-egalitarianism [58].

Societal factors affect not only a population's average egalitarianism, but also the difference in egalitarianism between groups occupying different positions on the societal hierarchy. The largest cross-national metaanalysis of SDO found that although, as predicted by social dominance theory [2], average anti-egalitarianism levels are always greater among men (versus women) and high power (versus low power) ethnic groups, the degree of dissociation in equality concerns between the groups depends on national and cultural context. Those countries with greater economic wealth, higher levels of gender equality, and more individualistic and liberal values exhibit greater differences in SDO between gender and ethnic groups than countries that are less advanced in terms of economic development and gender empowerment, and more traditional and collectivistic in values [51]. This suggests that as a society becomes more modern, its citizens are more likely to adjust their relational expectations away from a collective consensus (whether concerning equality or hierarchy), to fit with the interests of their specific group.

Finally, societal characteristics also affect the way in which equality (versus hierarchy) is implemented in the context of intergroup relations, as reflected in the predictive power of egalitarianism vis-à-vis other social and political attitudes. Just as a population's average level, as well as its differential group-based patterning, of egalitarianism increases as its social ecology progresses towards equality on the parameters above, so does the formation of sociopolitical attitudes by egalitarianism: Stronger negative correlations between SDO and support for the poor, protection of minorities, support for women in leadership, and environmentalism, are observed in countries with less economic distress, greater democratic protections, greater gender empowerment, and better environmental standards, respectively $[59,60]$. This pattern mirrors the finding at the within-nation level that having more economic resources heightens egalitarianism's predictive power [34], in line with social dominance theory's claim that ideological coherence is stronger among high power groups [2]. The overall pattern suggests that as severe ecological constraints are lifted, people will draw more strongly on their underlying relational orientation in deciding attitudes towards the distribution of resources among groups in society.

Of course, an interactionist perspective implies that the relationship between individual levels of egalitarianism and socio-ecological experiences is bidirectional and complex. Just as socio-ecology shapes the dynamics of equality concerns, so socio-ecological cues are mediated through relational preferences. Indeed, egalitarianism shapes how one experiences personal discrimination [33], material signs of inequality [61], and exposure to others in need [62,63]. A recent meta-analysis of 660 samples across 36 cultures found that cultures with more egalitarian (versus hierarchical) values produced stronger associations between intergroup contact and prejudice [64], implying that positive social experiences can only fully translate into improved social attitudes in a cultural context supportive of equality as the principle governing societal resource distribution. 


\section{Conclusion \& outstanding issues}

Understanding egalitarianism in terms of fundamental relational motives manifesting in socio-ecological context enables the drawing of connections across disciplines and levels of analysis. The developmental literature points toward an early-emerging understanding of the implications of equality and hierarchy for the distribution of resources and rights, and possibly stable preferences for the application of these relational models to contexts of resource distribution. These basic preferences are likely precursors to later, enduring individual differences in the preferred application and implementation of equality versus hierarchy to the context of societal intergroup relations, as uncovered by political psychologists. The question of how and when individuals gravitate toward one or the other relational model in adopting political attitudes draws on multiple factors. Inherited characteristics such as sex, physical formidability, and temperament should interact with the experience of being socialized into a particular role and group, in addition to the wider societal dynamics concerning power, resources, and environmental conditions (see also [65]).

Fully fleshing out this picture will entail empirical efforts with just such a transdisciplinary, multi-level sensibility. For example, the question of what occurs between early childhood and adulthood could be addressed with longitudinal studies tracking how experiences such as family socioeconomic status, parenting style, and school playground dynamics shape the relational orientations of young people as they mature. Multilevel analyses across towns, provinces, and countries can help reveal how institutional policies and political systems affect the evolving attitudes of particular population segments toward the most divisive political questions concerning who is entitled to what. Addressing these questions with an eye to both the psychological potency of social-structural conditions and the intuitive relational logic that evolved through species and generations will enable a full account of egalitarianism as a classic socio-ecological system.

\section{References and recommended reading}

Papers of particular interest, published within the period of review, have been highlighted as:

* of special interest

** of outstanding interest

[1] A.P. Fiske, Structures of social life: The four elementary forms of human relations: Communal sharing, authority ranking, equality matching, market pricing, Free Press, New York, NY, US, 1991.

[2] J. Sidanius, F. Pratto, Social Dominance: An Intergroup Theory of Social Hierarchy and Oppression, Cambridge University Press, New York, NY, US, 1999.

[3] B. Franks, Social construction, evolution and cultural universals, Cult. Psychol. 20 (2014) 416-439. doi:10.1177/1354067X14542524.

[4] L. Thomsen, S. Carey, Core cognition of social relations, Navig. Soc. World What Infants Child. Species Can Teach Us. (2013) 1-14.

[5] J.A. Sommerville, E.A. Enright, The origins of infants' fairness concerns and links to prosocial behavior, Curr. Opin. Psychol. 20 (2018) 117-121. doi:10.1016/j.copsyc.2018.01.005.

[6] M. Buyukozer Dawkins, S. Sloane, R. Baillargeon, Do Infants in the First Year of Life Expect Equal Resource Allocations?, Front. Psychol. 10 (2019). doi:10.3389/fpsyg.2019.00116.

[7] K. Lucca, J. Pospisil, J.A. Sommerville, Fairness informs social decision making in infancy, PLOS ONE. 13 (2018) e0192848. doi:10.1371/journal.pone.0192848.

[8] F. Margoni, L. Surian, Infants' evaluation of prosocial and antisocial agents: A meta-analysis., Dev. Psychol. 54 (2018) 1445-1455. doi:10.1037/dev0000538.

[9] * T. Ziv, J.A. Sommerville, Developmental differences in infants' fairness expectations from 6 to 15 months of age, Child Dev. 88 (2017) 1930-1951. doi:10.1111/cdev.12674.

This paper replicates earlier studies in providing evidence of a basic form of individual differences in egalitarianism in infancy, while also showing the role of age and experience in the onset of egalitarian expectations. Results from a series of studies support a developmental shift at around 9 months of age in the detection of equal versus unequal outcomes between novel agents, which depends on the onset of naturalistic sharing as reported by parents, and the presence of siblings. At 12 and 15 months, individual differences in infants' sensitivity to inequality were associated with their willingness to share a preferred toy with an experimenter, implying that proto-egalitarianism is linked to altruism in the early years. 
[10] * E.A. Enright, H. Gweon, J.A. Sommerville, 'To the victor go the spoils': Infants expect resources to align with dominance structures, Cognition. 164 (2017) 8-21.

doi:https://doi.org/10.1016/j.cognition.2017.03.008.

Through a set of looking time studies with 17-month-olds, this paper combines the paradigms investigating core cognition of equality and dominance, showing how the two are intertwined. Building on prior findings that infant expectations of equality depend on relative merit (Sloane et al., 2012), and that their expectations regarding dominance extend to resource conflict (Mascaro \& Csibra, 2012), the authors report that the expectation of equal resource distribution between two agents varies with information about their dominance. Once infants have seen evidence that one of two agents is dominant through watching the outcome of a conflicting goals scenario, they look longer to an equal distribution outcome, or one in which the submissive agent receives more, than to an outcome in which the dominant agent receives more. This provides evidence among preverbal infants that although expectations of equal resource distribution may be the default, the introduction of a dominance hierarchy immediately solicits the expectation that windfall resources will be unequally distributed to its parties, depending on status. In other words, for infants and arguably also for adults, what is expected or seen as 'right' for a particular context of resource distribution depends on cues regarding whether equality or hierarchy is the most appropriate relational model in that context.

[11] A. Guinote, I. Cotzia, S. Sandhu, P. Siwa, Social status modulates prosocial behavior and egalitarianism in preschool children and adults, Proc. Natl. Acad. Sci. 112 (2015) 731-736. doi:10.1073/pnas.1414550112.

[12] L. Thomsen, W.E. Frankenhuis, M. Ingold-Smith, S. Carey, Big and Mighty: Preverbal Infants Mentally Represent Social Dominance, Science. 331 (2011). doi:10.1126/science.1199198.

[13] O. Mascaro, G. Csibra, Representation of stable social dominance relations by human infants, Proc. Natl. Acad. Sci. 109 (2012) 6862. doi:10.1073/pnas.1113194109.

[14] A. Pun, S.A.J. Birch, A.S. Baron, Infants use relative numerical group size to infer social dominance, Proc. Natl. Acad. Sci. 113 (2016) 2376. doi:10.1073/pnas.1514879113.

[15] * A.J. Thomas, L. Thomsen, A.F. Lukowski, M. Abramyan, B.W. Sarnecka, Toddlers prefer those who win but not when they win by force, Nat. Hum. Behav. 2 (2018) 662. doi:10.1038/s41562-018-0415-3.

This paper provides the first evidence that young toddlers selectively prefer and approach those who prevail in zero-sum conflicts, suggesting an early-developing motive to affiliate with others of high rank, though one that is conditional on the voluntary submission of others. Toddlers aged 21-31 months chose a puppet who had prevailed over a puppet who yielded in the in the conflicting goals, right-of-way scenario of Thomsen et al (2011) by prostrating and moving away from the other. However, when the dominant agent prevailed simply by using force, toddlers avoided it. This implies an early emerging preference for those at the top of dominance hierarchies, but only if their status is recognized and yielded to.

[16] M.F.H. Schmidt, J.A. Sommerville, Fairness Expectations and Altruistic Sharing in 15-Month-Old Human Infants, PLOS ONE. 6 (2011) 1-7. doi:10.1371/journal.pone.0023223.

[17] S. Sloane, R. Baillargeon, D. Premack, Do infants have a sense of fairness?, Psychol. Sci. 23 (2012) 196-204. doi:10.1177/0956797611422072.

[18] E. Fehr, H. Bernhard, B. Rockenbach, Egalitarianism in young children, Nature. 454 (2008) 10791083. doi:10.1038/nature07155.

[19] L. Bian, S. Sloane, R. Baillargeon, Infants expect ingroup support to override fairness when resources are limited., Proc. Natl. Acad. Sci. U. S. A. 115 (2018) 2705-2710. doi:10.1073/pnas.1719445115.

[20] F. Pratto, J. Sidanius, L. Stallworth, B. Malle, Social Dominance Orientation, J. Pers. Soc. Psychol. 67 (1994) 741-763.

[21] A.K. Ho, J. Sidanius, F. Pratto, S. Levin, L. Thomsen, N. Kteily, J. Sheehy-Skeffington, Social Dominance Orientation: Revisiting the Structure and Function of a Variable Predicting Social and Political Attitudes, Pers. Soc. Psychol. Bull. 38 (2012) 583-606. doi:10.1177/0146167211432765.

[22] A.K. Ho, J. Sidanius, N. Kteily, J. Sheehy-Skeffington, F. Pratto, K.E. Henkel, R. Foels, A.L. Stewart, The nature of social dominance orientation: Theorizing and measuring preferences for intergroup inequality using the new $\mathrm{SDO}_{7}$ scale, J. Pers. Soc. Psychol. 109 (2015) 1003-1028. doi:10.1037/pspi0000033.

[23] F. Pratto, J. Sidanius, S. Levin, Social dominance theory and the dynamics of intergroup relations: Taking stock and looking forward, Eur. Rev. Soc. Psychol. 17 (2006) 271-320. doi:10.1080/10463280601055772. 
[24] J. Sidanius, S. Cotterill, J. Sheehy-Skeffington, N.S. Kteily, H. Carvacho, Social dominance theory: Explorations in the psychology of oppression., in: C.G. Sibley, F.K. Barlow (Eds.), Camb. Handb. Psychol. Prejudice, 2016: pp. 149-187.

[25] P.T. Dunwoody, S.G. McFarland, Support for Anti-Muslim Policies: The Role of Political Traits and Threat Perception, Polit. Psychol. 39 (2018) 89-106. doi:10.1111/pops.12405.

[26] C. Scott, S. Safdar, Threat and Prejudice against Syrian Refugees in Canada: Assessing the Moderating Effects of Multiculturalism, Interculturalism, and Assimilation, Int. J. Intercult. Relat. 60 (2017) 2839. doi:10.1016/j.ijintrel.2017.06.003.

[27] J.V. Assche, K. Dhont, T.F. Pettigrew, The social-psychological bases of far-right support in Europe and the United States, J. Community Appl. Soc. Psychol. 0 (n.d.). doi:10.1002/casp.2407.

[28] D.C. Mutz, Status threat, not economic hardship, explains the 2016 presidential vote, Proc. Natl. Acad. Sci. 115 (2018) E4330-E4339. doi:10.1073/pnas.1718155115.

[29] J. Womick, T. Rothmund, F. Azevedo, L.A. King, J.T. Jost, Group-Based Dominance and Authoritarian Aggression Predict Support for Donald Trump in the 2016 U.S. Presidential Election, Soc. Psychol. Personal. Sci. (2018) 1948550618778290. doi:10.1177/1948550618778290.

[30] L. Thomsen, E.G.T. Green, J. Sidanius, We will hunt them down: How social dominance orientation and right-wing authoritarianism fuel ethnic persecution of immigrants in fundamentally different ways, J. Exp. Soc. Psychol. 44 (2008) 1455-1464. doi:10.1016/j.jesp.2008.06.011.

[31] * C. Bratt, J. Sidanius, J. Sheehy-Skeffington, Shaping the Development of Prejudice: Latent Growth Modeling of the Influence of Social Dominance Orientation on Outgroup Affect in Youth, Pers. Soc. Psychol. Bull. 42 (2016) 1617-1634. doi:10.1177/0146167216666267.

This paper provides the strongest existing evidence of egalitarianism as a stable trait which predicts inequality-relevant social attitudes over time. Cross-lagged latent growth curve modelling conducted on multi-wave samples of white Norwegian adolescents and American college students demonstrated substantial rank-order stability in social dominance orientation and evidence for its over-time influence on the development of prejudice toward other ethnic groups.

[32] N.S. Kteily, J. Sidanius, S. Levin, Social dominance orientation: Cause or 'mere effect'?, J. Exp. Soc. Psychol. 47 (2011) 208-214. doi:10.1016/j.jesp.2010.09.009.

[33] L. Thomsen, E.G.T. Green, A.K. Ho, S. Levin, C. van Laar, S. Sinclair, J. Sidanius, Wolves in Sheep's Clothing: SDO Asymmetrically Predicts Perceived Ethnic Victimization Among White and Latino Students Across Three Years, Pers. Soc. Psychol. Bull. 36 (2010) 225-238. doi:10.1177/0146167209348617.

[34] A. Azar, L. Maldonado, J.C. Castillo, J. Atria, Income, egalitarianism and attitudes towards healthcare policy: a study on public attitudes in 29 countries, Public Health. 154 (2018) 59-69. doi:10.1016/j.puhe.2017.09.007.

[35] R. Fischer, K. Hanke, C.G. Sibley, Cultural and Institutional Determinants of Social Dominance Orientation: A Cross-Cultural Meta-Analysis of 27 Societies, Polit. Psychol. 33 (2012) 437-467. doi:10.1111/j.1467-9221.2012.00884.x.

[36] T.H. Kleppesto, N.H. Eftedal, L. Thomsen, Social Dominance Orientation, Encycl. Evol. Psychol. Sci. (2021).

[37] C. Reyna, Scale creation, use, and misuse: How politics undermines measurement., in: J.T. Crawford, L. Jussim (Eds.), Polit. Soc. Psychol., Routledge, New York, NY, US, 2018.

[38] J. Tooby, L. Cosmides, On the Universality of Human Nature and the Uniqueness of the Individual: The Role of Genetics and Adaptation, J. Pers. 58 (1990) 17-67. doi:10.1111/j.14676494.1990.tb00907.x.

[39] M.B. Petersen, Laustsen, Lasse, Upper-Body Strength and Political Egalitarianism: Twelve Conceptual Replications, Polit. Psychol. 40 (2019) 375-394. doi:10.1111/pops.12505.

[40] M.B. Petersen, D. Sznycer, A. Sell, L. Cosmides, J. Tooby, The Ancestral Logic of Politics: UpperBody Strength Regulates Men's Assertion of Self-Interest Over Economic Redistribution, Psychol. Sci. 24 (2013) 1098-1103. doi:10.1177/0956797612466415.

[41] M.E. Price, J. Kang, J. Dunn, S. Hopkins, Muscularity and attractiveness as predictors of human egalitarianism, Personal. Individ. Differ. 5 (2011) 636-640. doi:10.1016/j.paid.2010.12.009.

[42] * M.E. Price, J. Sheehy-Skeffington, J. Sidnaius, N. Pound, Is sociopolitical egalitarianism related to bodily and facial formidability in men?, Evol. Hum. Behav. 38 (2017) 626-634. 
This paper sheds light on one way in which individual levels of egalitarianism draw on enduring embodied characteristics and aspects of the socio-ecological context. It adds to the evidence base regarding the association between anti-egalitarianism and physical formidability especially among high income males, while also showing that this holds controlling for time invested in increasing physical formidability.

[43] R.C. Arslan, L. Penke, Evolutionary Genetics, in: Handb. Evol. Psychol., American Cancer Society, 2015: pp. 1-20. doi:10.1002/9781119125563.evpsych245.

[44] D. Nettle, The evolution of personality variation in humans and other animals., Am. Psychol. 61 (2006) 622-631. doi:10.1037/0003-066X.61.6.622.

[45] J.M. Smith, Evolution and the Theory of Games, Cambridge University Press, 1982.

[46] ** T.H. Kleppesto, N. Czajkowski, O. Vassend, E. Roysamb, Eftedal, J. Sheehy-Skeffington, J.R. Kunst, L. Thomsen, Correlations Between Social Dominance Orientation and Political Attitudes Reflect Common Genetic Underpinnings: A Twin Study, Proc. Natl. Acad. Sci. (In press). https://doi.org/10.31219/osf.io/9bfaj.

This study provides the first evidence that egalitarianism and its association with political attitudes is in part genetically grounded. Multivariate behavioural genetics analysis of a sample of almost 2000 Norwegian twins revealed $37 \%$ heritability in the dominance sub-dimension of social dominance orientation and $24 \%$ heritability in the equality sub-dimension. The genetic correlation between these sub-dimensions and political attitudes was overall high (mean genetic correlation 0.51 ) and the environmental correlation was very low (mean environmental correlation 0.08). The largest factor underpinning SDO was unique environmental experiences and there was no discernable effect of shared family environment, pointing to the importance of adult social and societal experiences on egalitarianism as reported in late middle age. Although there was a strong unique environment effect on SDO, its covariation with political attitudes was primarily genetic. The results are thus consistent with the existence of a heritable behavioral syndrome linking the preferred application of the relational model of hierarchy versus equality to societal intergroup relations with its implementation through whatever hierarchy-enhancing versus hierarchy-attenuating means the socio-ecological context affords.

[47] M. Wolf, F.J. Weissing, Animal personalities: consequences for ecology and evolution, Trends Ecol. Evol. 27 (2012) 452-461. doi:10.1016/j.tree.2012.05.001.

[48] C. Meeusen, K. Dhont, Parent-Child Similarity in Common and Specific Components of Prejudice: The Role of Ideological Attitudes and Political Discussion, Eur. J. Personal. 29 (2015) 585-598. doi:10.1002/per.2011.

[49] D. Nettle, K. Panchanathan, T.S. Rai, A.P. Fiske, The Evolution of Giving, Sharing, and Lotteries, Curr. Anthropol. 52 (2011) 747-756. doi:10.1086/661521.

[50] D. Nettle, R. Saxe, Preferences for redistribution are sensitive to perceived luck, social homogeneity, war and scarcity, (2019). doi:10.31234/osf.io/kupqv.

[51] I.-C. Lee, F. Pratto, B.T. Johnson, Intergroup Consensus/Disagreement in Support of Group Based Hierarchy: An Examination of Socio-Structural and Psycho-Cultural Factors, Psychol. Bull. 137 (2011) 1029-1064. doi:10.1037/a0025410.

[52] S. Guimond, M. Dambrun, N. Michinov, S. Duarte, Does social dominance generate prejudice? Integrating individual and contextual determinants of intergroup cognitions, J. Pers. Soc. Psychol. 84 (2003) 697-721.

[53] M. Li, Priming Mediated Vicarious Intergroup Contact: How Narrative Focus Influences Attitude Changes Toward Gay People, Same-Sex Family, and Social Dominance, Imagin. Cogn. Personal. (2018) 0276236618810203. doi:10.1177/0276236618810203.

[54] E. Huppert, J.M. Cowell, Y. Cheng, C. Contreras-Ibáñez, N. Gomez-Sicard, M.L. Gonzalez-Gadea, D. Huepe, A. Ibanez, K. Lee, R. Mahasneh, S. Malcolm-Smith, N. Salas, B. Selcuk, B. Tungodden, A. Wong, X. Zhou, J. Decety, The development of children's preferences for equality and equity across 13 individualistic and collectivist cultures, Dev. Sci. 22 (2019) e12729. doi:10.1111/desc.12729.

[55] P.R. Blake, K. McAuliffe, J. Corbit, T.C. Callaghan, O. Barry, A. Bowie, L. Kleutsch, K.L. Kramer, E. Ross, H. Vongsachang, R. Wrangham, F. Warneken, The ontogeny of fairness in seven societies, Nature. 528 (2015) 258-261. doi:10.1038/nature15703.

[56] K. McAuliffe, P.R. Blake, N. Steinbeis, F. Warneken, The developmental foundations of human fairness, Nat. Hum. Behav. 1 (2017) 0042. doi:10.1038/s41562-016-0042.

[57] J. Henrich, S.J. Heine, A. Norenzayan, The weirdest people in the world?, Behav. Brain Sci. 33 (2010) 61-83. doi:10.1017/S0140525X0999152X. 
[58] ** J.R. Kunst, R. Fischer, J. Sidanius, L. Thomsen, Preferences for group dominance track and mediate the effects of macro-level social inequality and violence across societies, Proc. Natl. Acad. Sci. 114 (2017) 5407-5412. doi:10.1073/pnas.1616572114.

This paper provides evidence for multilevel influences on and effects of egalitarianism across socioecological contexts. Cross-nationally $(n=41,824)$, lower egalitarianism, measured in terms of higher social dominance orientation (SDO), was observed among dominant groups in countries with greater gender and happiness inequality and lower democracy, governance, press freedom, and social progress. A similar pattern was found among White Americans across U.S. states $(n=4,613)$, with their average levels of SDO being positively correlated with income inequality and risk of violence. Multilevel analyses revealed that individual levels of SDO mediate the impact of state-level inequality and violence on downstream social attitudes including supporting the persecution of immigrants, racism, sexism, and opposing social welfare. Thus, while macro-structural factors shape average levels of egalitarianism in a population, its meaningful covariation with political attitudes occurs at the individual level, consistent with the notion of a convergent adaptive strategy that is in part set by socio-ecological conditions, and in turn predicts support for specific policies and ideologies that enhance or attenuate social hierarchy.

[59] T.L. Milfont, P.G. Bain, Y. Kashima, V. Corral-Verdugo, C. Pasquali, L.-O. Johansson, Y. Guan, V.V. Gouveia, R.B. Garðarsdóttir, G. Doron, M. Bilewicz, A. Utsugi, J.I. Aragones, L. Steg, M. Soland, J. Park, S. Otto, C. Demarque, C. Wagner, O.J. Madsen, N. Lebedeva, R. González, P.W. Schultz, J.L. Saiz, T. Kurz, R. Gifford, C.S. Akotia, N.M. Saviolidis, G. Einarsdóttir, On the Relation Between Social Dominance Orientation and Environmentalism: A 25-Nation Study, Soc. Psychol. Personal. Sci. 9 (2018) 802-814. doi:10.1177/1948550617722832.

[60] F. Pratto, A. Çidam, A.L. Stewart, F.B. Zeineddine, M. Aranda, A. Aiello, X. Chryssochoou, A. Cichocka, J.C. Cohrs, K. Durrheim, V. Eicher, R. Foels, P. Górska, I.-C. Lee, L. Licata, J.H. Liu, L. Li, I. Meyer, D. Morselli, O. Muldoon, H. Muluk, S. Papastamou, I. Petrovic, N. Petrovic, G. Prodromitis, F. Prati, M. Rubini, R. Saab, J. van Stekelenburg, J. Sweetman, W. Zheng, K.E. Henkel, Social Dominance in Context and in Individuals: Contextual Moderation of Robust Effects of Social Dominance Orientation in 15 Languages and 20 Countries, Soc. Psychol. Personal. Sci. 4 (2013) 587599. doi:10.1177/1948550612473663.

[61] * N. Kteily, J. Sheehy-Skeffington, A. Ho, Hierarchy in the Eye of the Beholder, J. Pers. Soc. Psychol. 112 (2017) 136-159. doi:10.1037/pspp0000097.

Complementing evidence for how egalitarianism is underpinned by a basic sensitivity to inequality, this paper's findings suggest the reverse process: how sensitivity to inequality, this time at a societal scale, is shaped by egalitarianism. Eight studies demonstrate that those higher in anti-egalitarianism, as measured by social dominance orientation, are less likely to notice inequality in their society, in fictitious societies, and even in abstract images, and, that this mediates lower support for egalitarian social policies. This result holds even where participants are incentivized to report accurately and when asked about memory for inequality, implying that it is a case of motivated perception operating below the level of conscious awareness.

[62] B. Lucas, N. Kteily, (Anti-)Egalitarianism Differentially Predicts Empathy for Members of Advantaged Versus Disadvantaged Groups, J. Pers. Soc. Psychol. 114 (2018) 665-692. doi:10.1037/pspa0000112.

[63] J. Sidanius, N. Kteily, J. Sheehy-Skeffington, A.K. Ho, C. Sibley, B. Duriez, You're inferior and not worth our concern: the interface between empathy and social dominance orientation., J. Pers. 81 (2013) 313-323. doi:10.1111/jopy.12008.

[64] J. Kende, K. Phalet, W. Van den Noortgate, A. Kara, R. Fischer, Equality Revisited: A Cultural MetaAnalysis of Intergroup Contact and Prejudice, Soc. Psychol. Personal. Sci. 9 (2018) 887-895. doi:10.1177/1948550617728993.

[65] J. Sheehy-Skeffington, L. Thomsen, A theory of how evolved psychology underpins attitudes towards societal economics must go beyond exchanges and averages, Behav. Brain Sci. 41 (2018).

\section{Acknowledgements}

This research was part-supported by Early Career Group Leader Grants 0602-01839B and 231157/F10 from the Independent Research Fund Denmark and the Norwegian Research Council, respectively (to L.T.). 